\title{
The SETDB2 locus: evidence for a genetic link between handedness and atopic disease
}

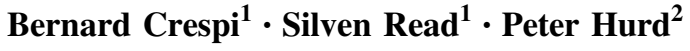

Received: 25 May 2017 / Revised: 8 August 2017 / Accepted: 9 August 2017 / Published online: 10 November 2017

(C) The Genetics Society 2018

\begin{abstract}
The gene SETDB2, which mediates aspects of laterality in animal model systems, has recently been linked with human handedness as measured continuously on a scale from strong left to strong right. By contrast, it was marginally associated with a left-right dichotomous measure, and it showed no evidence of association with absolute handedness strength independent of direction. We genotyped the SETDB2 handedness-associated single nucleotide polymorphism, rs4942830, in a large healthy population likewise phenotyped for continuous, absolute, and dichotomous handedness variables. Our results demonstrated significant effects of rs4942830 genotype on continuous handedness, and weaker, marginal effects on dichotomous handedness, but no effects on absolute handedness. These results help to establish the locus marked by the SNP rs4942830 as a strong candidate for mediating human handedness. Intriguingly, rs 4942830 is also in complete linkage disequilibrium with rs386770867, a polymorphism recently shown to affect human serum levels of IgE production and other atopic phenotypes. These findings implicate this locus in the longstanding links of handedness with asthma and other atopic diseases.
\end{abstract}

\section{Introduction}

Human handedness exhibits substantial heritability, on the order of $0.3-0.7$ depending on how it is measured (Lien et al. 2015). However, identification of specific genes that underlie variation in handedness has been challenging. Three genes, AR (Androgen Receptor), LRRTMI (Leucine Rich Repeat Transmembrane Neuronal 1), and PCSK6 (Proprotein Convertase Subtilisin/Kexin Type 6) stand out as strong candidates (Medland et al. 2005; Francks et al. 2007; Scerri et al. 2010; Hampson and Sankar 2012; Arning et al. 2013; Leach et al. 2014; Robinson et al. 2016). Even for these genes, however, replications have been inconsistent with regard to the specific markers implicated, the populations demonstrating associations (e. g., typical or with dyslexia), and the specific metrics of handedness associated with genetic or epigenetic variation. As a result, studies of the molecular and developmental genetic bases of

Bernard Crespi

crespi@sfu.ca

1 Department of Biological Sciences, 8888 University Drive, Simon Fraser University, Burnaby, British Columbia V5A 1S6, Canada

2 Department of Psychology and Centre for Neuroscience, University of Alberta, Edmonton T6G 2R3, Canada human handedness have been hindered by a lack of validated markers and candidate loci.

The gene SETDB2 (SET Domain Bifurcated 2) was recently associated with human handedness variation by Ocklenburg et al. 2016, in a Caucasian population. This gene represents a strong a priori functional candidate given that its gene product regulates left-right asymmetries in the central nervous system, at least in part though effects on expression of the growth factor fgf8 (fibroblast growth factor 8) (Xu et al. 2010). Ocklenburg et al. 2016 demonstrated that the SNP rs 4942830 was significantly associated with a continuous measure of handedness from strong left to strong right, but not associated with strength of handedness, and associated only nominally (not after multiplecomparison adjustment) with handedness categorized as left vs. right.

The SETDB2 locus is also of notable interest because its chromosomal location at $13 \mathrm{q} 14$ represents a well-validated genomic region for association with risk of atopic diseases (mainly asthma, eczema, and hay fever) and with serum levels of the immune molecule, IgE, that mediates atopic hypersensitivities (Wills-Karp and Ewart 2004; Jones and Stewart 2010; Holt et al. 2011, 2015). These findings suggest that genetic variation in this region may underlie, to some degree, the epidemiological connections of atopic disease with handedness (Geschwind and Behan 1982, 1984; Smith 1987; Weinstein and Pieper 1988; Tonnessen 
et al. 1993; Andreou et al. 2000; Kaynar and Dane 2003; Krommydas et al. 2003, 2004; Peters et al. 2006; Preti et al. 2008) that trace back to seminal work by Geschwind and Galaburda (1985). By the Geschwind-Galaburda hypothesis, which has remained controversial since its inception due to inconsistencies in evidence and ignorance of causal mechanisms (e.g., Bryden et al. 1994), handedness and immune disorders are associated due in part to their shared mediation by fetal testosterone levels. Testosterone has indeed been associated with aspects of atopic immune disorders (e.g., Shaheen et al. 2007), and measures of handedness (Hampson and Sankar 2012), but specific molecular and developmental bases for associations have yet to be identified. Discovery and analysis of such causal factors is important because they will provide novel insights into the workings of the human neuroimmune system, and its genetic, epigenetic and environmental underpinnings, and provide for robust tests and extensions of Geschwind and Galaburda's (1985) influential ideas.

This study has two goals. First, we set out to replicate the results of Ocklenburg et al. 2016, by genotyping rs4942830 for a population that was phenotyped for the same three measures of handedness (continuous left to right, strength, and dichotomous direction) and conducting the same analyses. Second, we used publicly-available human genetic data, and salient literature, to evaluate the hypothesis that the locus marked by rs4942830 mediates pleiotropy of handedness with risks and phenotypes of atopic disease.

\section{Methods}

\section{Study population}

The study population was a sample of Caucasian undergraduate students from both University of Alberta (Edmonton, Canada) and Simon Fraser University (Burnaby, Canada) $(N=808,275$ males, 533 females, mean age of 19 with $\mathrm{SD}=2$ ). The data collection was approved by Human Research Ethics at University of Alberta and the Simon Fraser University Research Ethics Board, and subjects provided written informed consent prior to participation.

\section{Handedness data}

Participants filled out the Waterloo Handedness Questionnaire (WHQ) (Steenhuis et al. 1990). Scores for each of 32 WHQ items ranging from +2 for strong right, +1 for weak right, 0 for ambidextrous, -1 for weak left and -2 for strong left, giving a total scale range from -64 to +64 . Our metrics of handedness were constructed to match those of Ocklenburg et al. 2016: Laterality Quotient (LQ), a continuous measure from -64 to +64 that thus incorporates both direction and strength of handedness; Strength of Handedness (ST), the absolute value of LQ; and Left-Right (LR), a dichotomous measure whereby -1 to -64 is designated left and +1 to +64 is right. We also analyzed the handedness data using statistical protocols corresponding to those in Ocklenburg et al. 2016, with $\chi^{2}$ tests ( 3 by 2 contingency tables) used for analyzing the LR data in relation to genotype, for directness of interpretation.

\section{Genetic data}

DNA was extracted from saliva using standard phenolchloroform protocols. Fluorophore-labeled primers for SETDB2 rs4942830 were used for TaqMan ${ }^{\circledR}$ genotyping on a Roche LightCycler® 96 Real-Time PCR machine. VIC and FAM fluorescence data were analyzed under Endpoint Genotyping using LightCycler® 96 software, version 1.1.0.1320. Genotypes were in Hardy-Weinberg equilibrium $\left(\chi^{2}=0.94, P=0.33\right)$.

\section{Results}

\section{Handedness}

The average LQ was $36(\mathrm{SD}=24)$ and average $\mathrm{ST}$ was 41 $(\mathrm{SD}=15)$. Overall, $9.3 \%$ of participants were classified as left handed and $90.7 \%$ as right handed, by the LR measure. The three handedness variables were all significantly correlated with one another (LQ-ST, $\mathrm{r}=0.652, P<0.0001$; LQ-LR, $\mathrm{r}=0.808, \quad P<0.0001$; LR-ST, $\mathrm{r}=0.328, \quad P<$ 0.0001).

Main effects of sex, and sex by rs4942830 genotype, were both non-significant $(P=0.083$ and 0.56 respectively), in analysis of LQ scores as a function of genotype and sex, so the sexes were pooled for analysis, as in Ocklenburg et al. 2016. LQ differed significantly between the three genotype groups (Table 1) with low $\eta^{2}$ values indicating small magnitudes of effect; essentially the same significance values were also obtained under dominant $(P=0.036)$ and recessive $(P=0.034)$ models. The genotypic effects thus appeared to be linear, with the genotypes AA, AT, and TT each separated by about a three point decrease in right shift of LQ (Table 1). In contrast to Ocklenburg et al. (2016), the T allele was associated with lower levels of LQ.

Handedness categorized as LR showed an apparent pattern of increasing percent left-handedness with numbers of $\mathrm{T}$ alleles (AA, 6.8\%; AT, 9.1\%; TT, 13.8\%), but these differences were marginally non-significant (Table 1). The measure ST, in contrast to LQ and LR, showed no evidence of any differences between genotypes (Table 1). 
Table 1 Tests for association of the three handedness metrics with SETDB2 rs4942830 genotype

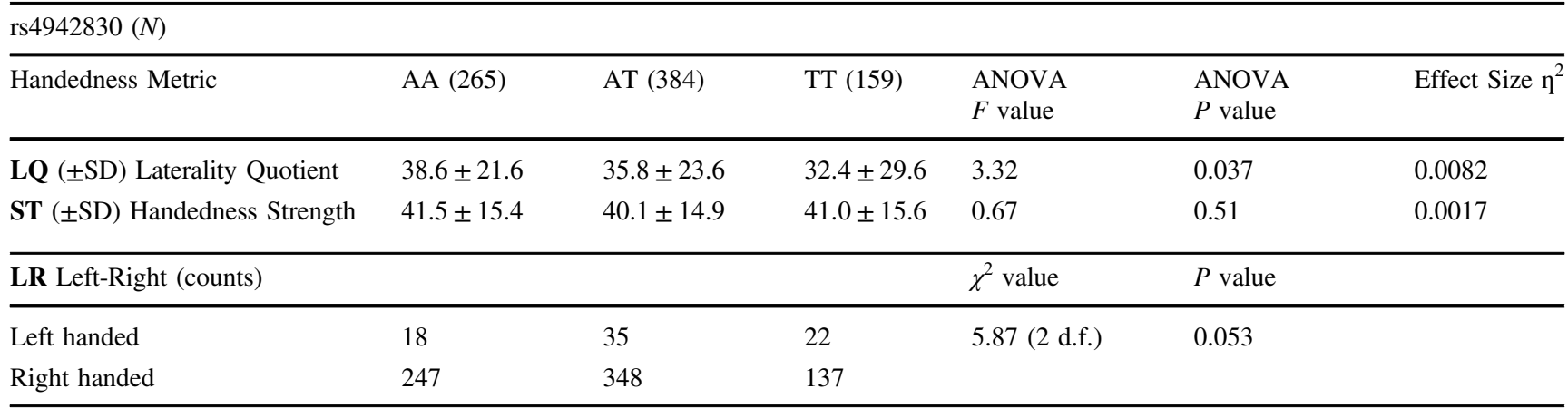

One individual had an WHQ score of zero and could not be categorized into left or right

\section{SETDB2 locus}

We used publically-available 1000 genomes phase 3 data to evaluate the hypothesis that the SETDB2 SNP rs4942830 marks a functional haplotype that mediates not just handedness, but also serum IgE and risks and phenotypes of atopic disease, given previous evidence of a QTL for serum IgE and asthma in this immediate region (Zhang et al. 2003; Wills-Karp and Ewart 2004).

The SNP rs4942830 is located in the first intron of the SETDB2 gene, about $6 \mathrm{~kb}$ from the start of its coding sequence. Multiple transcripts are produced from this region, including some that encompass just $S E T D B 2$, and one that extends to also include the gene PHF11 (PHD Finger Protein 11) (Zhang et al. 2003); this extended transcript is referred to as SETDB2-PHF11 (NCBI gene ID 107303344).

The two SNP markers in this region most strongly and directly linked with human IgE levels, and with risks and phenotypes of atopic disease, are rs386770867 (an AT/G indel polymorphism in the $5^{\prime}$ untranslated region of the SETDB2 gene) and rs1046295 (an A/G polymorphism in the $3^{\prime}$ untranslated region of the gene PHF11) (Holt et al. 2011, 2015).

In the CEU Caucasian population, rs386770867 (and its overlapping proxy SNP rs71307668) are in complete linkage disequilibrium (LD) $\left(\mathrm{D}^{\prime}=1\right.$ and $\left.\mathrm{R}^{2}=1\right)$ with rs4942830, and the two SNPs are separated by only 536 base pairs. LD values were virtually the same as in CEU in East Asian (CHB, JPT, CHS, CDX, KHV) and South Asian (GIH, PJL, BEB, STU, ITU) populations $\left(\mathrm{D}^{\prime}=0.99-1, \mathrm{R}^{2}\right.$ $=0.97-1$ ), as well as in the other Caucasian groups (TSI, FIN, GBR, IBS) (see Machiela and Chanock 2015, and https://analysistools.nci.nih.gov/LDlink for population codes). For the CEU data, rs386770867, rs71307668 and rs4942830 are all within the same haplotype block of about $9 \mathrm{~kb}$ in size (by Haploview), which comprises four haplotypes with frequencies of $0.58,0.26,0.10$ and 0.06 . In contrast to these populations, among Africans (YRI, LWK,
GWD, MSL, ESN), the markers rs386770867 and rs4942830 were not in linkage disequilibrium, with $\mathrm{D}^{\prime}=1$ but $\mathrm{R}^{2}=0.09-0.10$, apparently due to low frequency of the rs386770867 $\mathrm{G}$ allele in these populations (and the corresponding high frequency of AT). These results indicate that rs386770867 marks the same haplotype as rs4942830 among non-African human populations, rendering them essentially the same locus.

The IgE and atopy-linked SNP rs1046295, located within the PHF11 gene (and the SETDB2-PHF11 gene), is about 83 $\mathrm{kb}$ from rs4942830. Despite this distance, the two SNPs are in strong to moderate LD in the five European populations (overall, $\mathrm{D}^{\prime}=0.85, \mathrm{R}^{2}=0.68$ ), with lower $\mathrm{LD}$ in South Asians $\left(\mathrm{D}^{\prime}=0.84, \mathrm{R}^{2}=0.54\right)$, East Asians $\left(\mathrm{D}^{\prime}=0.62, \mathrm{R}^{2}=\right.$ $0.33)$, and Africans $\left(\mathrm{D}^{\prime}=0.80, \mathrm{R}^{2}=0.20\right)$ (populations same as above, and results are equivalent using rs386770867 instead of rs4942830). These results provide evidence that alleles of the SNPs rs4942830 and rs1046295 are notably associated with one another, especially for the European populations that have provided the bulk of the evidence regarding associations of the markers with $\operatorname{IgE}$ and atopic disease.

Ocklenburg et al. (2016) analyzed three SETDB2 SNPs in addition to rs4942830, none of which showed significant associations with handedness. If rs 4942830 shows the strongest association with both handedness and IgE/atopy, such that it marks a haplotype pleiotropically associated with both sets of phenotypes, then none of these other SNPs should be strongly linked with rs 4942830 or rs 1046295 . As expected, none of these SNPs are in strong LD with rs4942830 or rs1046295, in Europeans (CEU): rs4942830: highest $\mathrm{LD}$ with rs7998427, $\mathrm{D}^{\prime}=1, \mathrm{R}^{2}=0.56$; rs1046295: highest $\mathrm{LD}$ with rs7998427, $\mathrm{D}^{\prime}=0.97, \mathrm{R}^{2}=0.43$. The SNP rs7998427 also shows the strongest non-significant association with LQ in Ocklenburg et al. (2016), as expected given these patterns of LD.

Taken together, these results indicate that the handedness-associated SNP rs4942830 and the IgE/atopic disease-associated SNP rs386770867 mark the same genetic variation, providing evidence for pleiotropic effects on these 
two sets of phenotypes. Moreover, their substantial LD with the PHF11 SNP rs1046295 suggests the hypothesis that an extended haplotype, from the $5^{\prime}$ UTR of SETDB2 at least to the $3^{\prime}$ end of PHF11, may mediate both handedness and IgE/atopy risk variation. This hypothesis is consistent with the location of rs4942830 and rs386770867 $5^{\prime}$ upstream of SETDB2 and PHF11, the presence of a transcript that extends across the two genes in humans and mice (Zhang et al. 2003, 2014), and the documented effects of rs386770867 allelic variation on transcriptional activity as mediated by the transcription factors YY1 and SRY (Holt et al. 2015). The hypothesis requires direct testing, however, as multiple loci within these genes may certainly affect their expression or functional activity.

\section{Discussion}

This study contributes to the literature on the genetics of handedness and laterality in two main ways. First, the data analyzed here generally corroborates the results from Ocklenburg et al. (2016), in demonstrating significant association of allelic variation in the SETDB2 SNP rs4942830 with human handedness measured continuously from strong left to strong right (LQ). We also demonstrated, as did Ocklenburg et al. (2016), a complete lack of association of rs4942830 with handedness strength (ST) (independent of direction), and a marginally non-significant association with handedness dichotomized to left vs. right (LR). This latter, marginally non-significant result should, notwithstanding, be interpreted in the context of the close relationship between LQ and LR, and the loss of information and statistical power caused by pooling all individuals with LQ values above, or below, the midpoint. These findings establish the gene and haplotype marked by rs4942830 as a strong functional candidate for a 'handedness gene', as well as providing evidence for a genetic basis to the relationship of continuous handedness directionality with non-directional handedness strength (Prichard et al. 2013; Ocklenburg et al. 2014). Additional studies of the SETDB2 in relation to handedness measures are required to evaluate the direction of the allelic association, which differed between Ocklenburg et al. (2016) and our results; such findings are not uncommon in association studies and may be attributable to unobserved multilocus effects or other causes (Lin et al. 2007).

The results reported here are consistent with a polygenic basis for human handedness effects (Medland et al. 2009; McManus et al. 2013) involving direction and strength (Ocklenburg et al. 2016). Many previous physiological and behavioral studies have demonstrated effects of handedness strength independent of direction (e.g., Luders et al. 2010; Lyle et al. 2012), which makes these results for SETDB2 of interest in their clear absence of any effect on handedness strength. Additional effects from interacting, or independent, environmental factors such as testosterone (e.g., Hampson and Sankar 2012; Hollier et al. 2014) and other impacts, working through epigenetic effects (Ocklenburg et al. 2017), have yet to be evaluated for SETDB2. Further analysis of the nature of effects from handedness-related genes, which will allow more robust tests of alternative models, also requires validation of additional such genes beyond the small number recognized thus far.

The second main contribution of this study is the demonstration that the handedness-associated SNP rs4942830 is in complete or virtually-complete linkage disequilibrium (in non-Africans) with a SNP, rs386770867, that has been strongly associated with atopic disease and serum levels of IgE, from both genetic-association and functionalgenetic studies (Holt et al. 2011, 2015). In particular, the rs386770867 indel alleles AT and G differentially regulate expression of SETDB2 (and, apparently, PHF11), through their effects on binding of the transcription factors YY1 and SRY (Holt et al. 2015). The SNPs rs4942830 and rs386770867 are also in substantial LD (especially among Caucasians) with a locus in the downstream gene PHF11, rs1046295, that has also been shown to strongly affect risk of atopic disease and levels of IgE (Holt et al. 2011, 2015).

The complete linkage disequilibrium of rs 4942830 with rs386770867 provides the first evidence indicative of pleiotropy between handedness and atopic disease, such that the same locus appears to affect both. This result is notable given the well-established epidemiological associations of left or mixed handedness with asthma and other atopic diseases (cited above), that have hitherto lacked evidence regarding clear functional or genetic bases. Thus far, these associations have been considered mainly in the context of the classical Geschwind-Galaburda hypothesis, whereby, as noted above, levels of testosterone during brain development influence both handedness and functioning of the immune system (Geschwind and Galaburda 1985). Any role for testosterone in effects of the SETDB2-PHF11 locus remains conjectural at this point, although Holt et al. (2015) detected significant sex differences in SETDB2 expression due to rs386770867 allelic variation, apparently due to the male-limited expression of the transcription factor SRY. Although neither we, nor Ocklenburg et al. (2016), detected significant main effects of sex or sex by genotype interactions on handedness, in our analyses these effects were only marginally non-significant $(P=0.083$ for a sex effect), and rs4942830 associations with LQ and LR appeared to be stronger among females than males. These results suggest that this locus exerts sex-differential effects, although how testosterone might be involved remains unclear.

As noted above, SETDB2 codes for a methyltransferase that influences expression of $F G F 8$, whose gene product has been demonstrated to modulate left-right structural 
asymmetries in vertebrates ( $\mathrm{Xu}$ et al. 2010); this gene is also involved in chromosomal segregation (Falandry et al. 2010). The function of PHF11 is less well characterized, although it has demonstrated roles in T-cell activation and viability (Rahman et al. 2010) and DNA repair (Gong et al. 2017). The different functions of these genes suggest the possibility that, across an extended haplotype of SETDB2PHF11, different SNPs may independently influence handedness and $\operatorname{IgE}$ /atopy. Although this hypothesis is worth considering, it can provide no explanation for the epidemiological evidence, given that both handedness and atopy are each underlain by many genes of relatively small effect (Armour et al. 2014; Ober 2016).

The primary significance of these results taken together is that they provide a functional-genetic window into deciphering apparent pleiotropic effects in the development of human handedness, laterality, and immunity with special reference to atopic disease. As such, studies that collect detailed haplotype data on the SETDB2-PHF11 locus, in conjunction with measurements of handedness, brain laterality, immune functioning, serum IgE, and atopic disorders, should generate novel insights into both human development and disease. Of particular importance will be sequencing across the haplotype containing rs4942830 and rs386770867 (a SNP whose local region shows high levels of polymorphisms), and conducing haplotype-based tests of associations with atopic disease and handedness, which should help to identify the presence and nature of specific functional variation in this region and provide more-precise tests for pleiotropy and effects from specific alleles. More generally, other GWAS loci underlying serum IgE and atopic disease risks might also usefully be investigated for links with handedness, to test for pleiotropic effects from other regions of the human genome.

\section{DATA archiving}

Data available from the Dryad Digital Repository: http://dx. doi.org/10.5061/dryad.vb769

Acknowledgements We thank NSERC for financial support, and we are especially grateful to L. Arning for helpful comments and materials.

\section{Compliance with ethical standards}

Conflict of interest The authors declare no conflicts of interest.

\section{References}

Andreou G, Karapetsas A, Gourgoulianis KI, Molyvdas PA (2000) Left-handedness and inheritance of bronchial asthma. Percept Mot Skills 90(2):371-372
Armour JA, Davison A, McManus IC (2014) Genome-wide association study of handedness excludes simple genetic models. Heredity 112(3):221-225

Arning L, Ocklenburg S, Schulz S, Ness V, Gerding WM, Hengstler JG et al. (2013) PCSK6 VNTR polymorphism is associated with degree of handedness but not direction of handedness. PLoS One 8(6):e67251

Arning L, Ocklenburg S, Schulz S, Ness V, Gerding WM, Hengstler JG (2015). Handedness and the $X$ chromosome: the role of androgen receptor CAG-repeat length. Sci Rep 5:8325

Bryden MP, McManus IC, Bulmanfleming MB (1994) Evaluating the empirical support for the Geschwind-Behan-Galaburda model of cerebral lateralization. Brain Cognit 26(2):103-167

Falandry C, Fourel G, Galy V, Ristriani T, Horard B, Bensimon E et al. (2010) CLLD8/KMT1F is a lysine methyltransferase that is important for chromosome segregation. J Biol Chem 285 (26):20234-20241

Francks C, Maegawa S, Laurén J, Abrahams BS, Velayos-Baeza A, Medland SE et al. (2007) LRRTM1 on chromosome 2p12 is a maternally suppressed gene that is associated paternally with handedness and schizophrenia. Mol Psychiatry 12(12):1129-1139

Geschwind N, Behan P (1982) Left-handedness: association with immune disease, migraine, and developmental learning disorder. Proc Natl Acad Sci USA 79(16):5097-5100

Geschwind N, Galaburda AM (1985) Cerebral lateralization: biological mechanisms, associations, and pathology: I. A hypothesis and a program for research. Arch Neurol 42(5):428-459

Geschwind N, Behan PO (1984) Laterality, hormones, and immunity. In: Geschwind N, Galaburda AM (eds) Cerebral Dominance: The Biological Foundations. Harvard, Cambridge, MA, pp 211-224

Gong Y, Handa N, Kowalczykowski SC, de Lange T (2017) PHF11 promotes DSB resection, ATR signaling, and HR. Genes Devel 31(1):46-58

Hampson E, Sankar JS (2012) Hand preference in humans is associated with testosterone levels and androgen receptor gene polymorphism. Neuropsychologia 50(8):2018-2025

Hollier LP, Maybery MT, Keelan JA, Hickey M, Whitehouse AJ (2014) Perinatal testosterone exposure and cerebral lateralisation in adult males: Evidence for the callosal hypothesis. Biol Psychol 103:48-53

Holt RJ, Vandiedonck C, Willis-Owen SA, Knight JC, Cookson WO, Moffatt MF et al. (2015) A functional AT/G polymorphism in the 5 -untranslated region of SETDB2 in the IgE locus on human chromosome 13q14. Genes Immun 16(7):488-494

Holt RJ, Zhang Y, Binia A, Dixon AL, Vandiedonck C, Cookson WO et al. (2011) Allele-specific transcription of the asthma-associated PHD finger protein 11 gene (PHF11) modulated by octamerbinding transcription factor 1 (Oct-1). J Allergy Clin Immunol 127(4):1054-1062

Jones G, Stewart G (2010) Association of PHF11 polymorphisms with asthma and allergy. Thorax 65(7):659-660

Kaynar H, Dane S (2003) Prevalence of left-handedness among patients with different respiratory diseases. Int J Neurosci 113(10):1371-1377

Krommydas G, Gourgoulianis KI, Andreou G, Molyvdas PA (2003) Left-handedness in asthmatic children. Pediatr Allergy Immunol 14(3):234-237

Krommydas GC, Gourgoulianis KI, Raftopoulos V, Kotrotsiou E, Paralikas T, Agorogiannis G et al. (2004) Non-right-handedness and asthma. Allergy 59(8):892-893

Leach EL, Prefontaine G, Hurd PL, Crespi BJ (2014) The imprinted gene LRRTM1 mediates schizotypy and handedness in a nonclinical population. J Hum Genet 59(6):332-336 
Lien YJ, Chen WJ, Hsiao PC, Tsuang HC (2015) Estimation of heritability for varied indexes of handedness. Laterality 20(4):469-482

Lin PI, Vance JM, Pericak-Vance MA, Martin ER (2007) No gene is an island: the flip-flop phenomenon. Am $\mathrm{J}$ Hum Genet 80(3):531-538

Luders E, Cherbuin N, Thompson PM, Gutman B, Anstey KJ, Sachdev P, Toga AW (2010) When more is less: associations between corpus callosum size and handedness lateralization. Neuroimage 52(1):43-49

Lyle KB, Hanaver-Torrez SD, Hacklander RP, Edlin JM (2012) Consistency of handedness, regardless of direction, predicts baseline memory accuracy and potential for memory enhancement. J. Exp. Psychol.: Learning, Memory, and Cognition 38(1):187-193

Machiela MJ, Chanock SJ (2015) LDlink: a web-based application for exploring population-specific haplotype structure and linking correlated alleles of possible functional variants. Bioinformatics 31(21):3555-3557

McManus IC, Davison A, Armour JA (2013) Multilocus genetic models of handedness closely resemble single-locus models in explaining family data and are compatible with genome-wide association studies. Ann N Y Acad Sci 1288(1):48-58

Medland SE, Duffy DL, Spurdle AB, Wright MJ, Geffen GM, Montgomery GW et al. (2005) Opposite effects of androgen receptor CAG repeat length on increased risk of left-handedness in males and females. Behav Genet 35(6):735-744

Medland SE, Duffy DL, Wright MJ, Geffen GM, Hay DA, Levy F et al. (2009) Genetic influences on handedness: data from 25,732 Australian and Dutch twin families. Neuropsychol 47(2):330-337

Ober C (2016) Asthma genetics in the post-GWAS era. Ann Am Thoracic Soc 13(Supplement 1):S85-S90

Ocklenburg S, Arning L, Gerding WM, Hengstler JG, Epplen JT, Güntürkün $\mathrm{O}$ et al. (2016) Left-right axis differentiation and functional lateralization: a haplotype in the methyltransferase encoding gene SETDB2 might mediate handedness in healthy adults. Mol Neurobiol 53(9):6355-6361

Ocklenburg S, Beste C, Arning L (2014) Handedness genetics: considering the phenotype. Front Psychol 5:1300

Ocklenburg S, Schmitz J, Moinfar Z, Moser D, Klose R, Lor S et al. (2017) Epigenetic regulation of lateralized fetal spinal gene expression underlies hemispheric asymmetries. eLife 6:e22784

Peters M, Reimers S, Manning JT (2006) Hand preference for writing and associations with selected demographic and behavioral variables in 255,100 subjects: the $\mathrm{BBC}$ internet study. Brain Cogn 62(2):177-189

Preti A, Lai A, Serra M, Zurrida GG (2008) Mixed handedness prevails among children and adolescents with infantile asthma and diabetes. Pediatr Allergy Immunol 19(8):769-772

Prichard E, Propper RE, Christman SD (2013) Degree of handedness, but not direction, is a systematic predictor of cognitive performance. Front Psychol 4:98-103

Rahman N, Stewart G, Jones G (2010) A role for the atopy-associated gene PHF11 in T-cell activation and viability. Immun Cell Biol 88(8):817-824

Robinson KJ, Hurd PL, Read S, Crespi BJ (2016) The PCSK6 gene is associated with handedness, the autism spectrum and magical ideation in a non-clinical population. Neuropsychologia $84: 205-212$

Scerri TS, Brandler WM, Paracchini S, Morris AP, Ring SM, Talcott JB et al. (2010) PCSK6 is associated with handedness in individuals with dyslexia. Hum Mol Genet 20(3):508-614

Shaheen SO, Hines M, Newson RB, Wheeler M, Herrick DR, Strachan DP et al. (2007) Maternal testosterone in pregnancy and atopic outcomes in childhood. Allergy 62(1):25-32

Smith J (1987) Left-handedness: its association with allergic disease. Neuropsychologia 25(4):665-674

Steenhuis RE, Bryden MP, Schwartz M, Lawson S (1990) Reliability of hand preference items and factors. J Clin Exp Neuropsychol 12:921-930

Tonnessen FE, Løkken A, Høien T, Lundberg I (1993) Dyslexia, lefthandedness, and immune disorders. Arch Neurol 50(4):411-416

Weinstein RE, Pieper DR (1988) Altered cerebral dominance in an atopic population. Brain Behav Immun 2(3):235-241

Wills-Karp M, Ewart SL (2004) Time to draw breath: asthmasusceptibility genes are identified. Nat Rev Genet 5(5):376-387

$\mathrm{Xu}$ PF, Zhu KY, Jin Y, Chen Y, Sun XJ, Deng M et al. (2010) $S E T D B 2$ restricts dorsal organizer territory and regulates left-right asymmetry through suppressing fgf8 activity. Proc Natl Acad Sci USA 107(6):2521-2526

Zhang Y, Dean C, Chessum L, Nguyen D, Stewart M, Taylor M et al. (2014) Functional analysis of a novel ENU-induced PHD finger 11 (PHF11) mouse mutant. Mamm Genome 25(11-12):573-582

Zhang Y, Leaves NI, Anderson GG, Ponting CP, Broxholme J, Holt R et al. (2003) Positional cloning of a quantitative trait locus on chromosome 13q14 that influences immunoglobulin E levels and asthma. Nat Genet 34(2):181-186 